\title{
Diseño, Construcción y Fabricación de una Máquina Prototipo de Medición por Coordenadas
}

Leonardo de J. Mesa-Palacio ${ }^{1}$

Gabriel Calle-Trujillo ${ }^{2}$

Juan J. Arbeláez-Toro ${ }^{3}$

\section{Resumen}

Este trabajo se enmarca como un estudio de caso de carácter teórico-práctico. La verificación metrológica de las piezas tipo marco se realiza por medio de mesas de medición y calibres. Dicho procedimiento, requiere tiempo en el montaje y precisión en los calibres. La investigación busca diseñar y construir una máquina prototipo de medición de coordenadas (CMM) que contribuya a la verificación metrológica de piezas tipo marco de motocicleta. Partiendo de la determinación de la exactitud requerida en la fabricación de bastidores se selecciona la estructura de la CMM, se determinan las tolerancias geométricas aplicadas a la construcción del bastidor de la CMM, se analizan las cadenas dimensionales y los principios de funcionamiento del Sistema de Ajustes y Tolerancias, se manufacturan y construyen las piezas que conforman la CMM. Posteriormente, se diseña el sistema de recolección, verificación y visualización. Finalmente, se procede a la calibración de la CMM, permitiendo conocer el error e incertidumbre de la CMM. El procedimiento descrito garantiza el diseño, construcción y fabricación de una máquina prototipo de medición por coordenadas tipo puente capaz de medir una longitud máxima de $250 \mathrm{~mm}$ con una tolerancia mínima de $\pm 0.3 \mathrm{~mm}$.

1 Facultad de Ingenierías, INSTITUTO TECNOLÓGICO METROPOLITANO, Medellín-Colombia, leonardomesa@itm.edu.co

2 Facultad de Ingeniería Mecánica, Universidad Tecnológica de Pereira, PereiraColombia, gcalle@utp.edu.co

3 Facultad de Ingenierías, INSTITUTO TECNOLÓGICO METROPOLITANO, Medellín-Colombia, juanarbelaez@itm.edu.co

Fecha de recepción: 13 de Agosto de 2010

Fecha de aceptación: 09 de Junio de 2011 


\section{Palabras clave}

Diseño, construcción, fabricación, máquina de medición por coordenadas.

\section{Abstract}

This work is framed as a case study of theoretical and practical. The metrological verification of frame parts type style is done through measurements and calibrations tables. This procedure requires time in the assembly and precision gauges. The research aims to design and build a prototype machine coordinate measurement (CMM) to assist in the verification metrology frame type of motorcycle parts. Based on the determination of the accuracy required in the manufacture of frames: select the structure of the CMM is selected, the geometric tolerances applied to the construction of the frame of the CMM are defined, the dimensional chains and the principles of operation of the system settings and tolerances are analyzed, are manufactured and build the pieces that conform the CMM are manufactured and built. Subsequently, the gathering, verification and display system is designed. Finally, we proceed to the calibration of the CMM, allowing identifying the error and uncertainty of the CMM. The procedure ensures design, construction, and manufacturing of a prototype machine type coordinate measuring bridge capable of measuring a maximum length of $250 \mathrm{~mm}$ with a minimum tolerance of $\pm 0.3 \mathrm{~mm}$.

\section{Keywords}

Design, construction, manufacture, coordinate measuring machine. 


\section{INTRODUCCIÓN}

En la fabricación de las piezas tipo marco de motocicleta, la verificación metrológica es realizada en mesas de medición con calibres diseñados específicamente para los diferentes subtipos de las piezas tipo marco. A pesar de ser un procedimiento eficiente, requiere de un permanente control sobre los calibres, los cuales deben ser verificados (dimensionalmente) y ajustados regularmente.

Otro procedimiento para la verificación se realiza mediante dispositivos como máquinas de medición por coordenadas de brazo articulado. Este garantiza un mejor control (en términos de confiabilidad de los resultados), pero a la vez implica una preparación más rigurosa en el montaje de las piezas, adicionando problemas como los saltos de los puntos de referencia y el tiempo de revisión (introduciendo otros aspectos a considerar como son los costos del equipo metrológico). Se propone entonces, el diseño, construcción y fabricación de una máquina de medición por coordenadas, que permita la verificación rápida y efectiva de las piezas tipo marco de motocicleta.

Esta investigación se inicia con la revisión bibliográfica de las diferentes máquinas de medición de coordenadas (CMM), se estudian sus aplicaciones y configuraciones; se analizan las tolerancias dimensionales de un bastidor de motocicleta tipo turismo (VIVA 115) y extraen las tolerancias más exigentes, las cuales ofrecen el punto de partida de la construcción de la CMM.

Partiendo de la exactitud requerida en la fabricación de bastidores de motocicleta, se realiza la selección de la configuración de la estructura de la CMM, desde el punto de vista de capacidad volumétrica, rigidez y funcionabilidad de la estructura. A continuación, se determinan las tolerancias geométricas aplicadas a la construcción del bastidor de la CMM que garantizan la exactitud global de las mediciones que se realizan en ella, pasando por el análisis de las cadenas dimensionales y los principios de funcionamiento del SAT (Sistema de Ajustes y Tolerancias).

Iniciando la fase de construcción, se analizan y se eligen los diferentes procesos de manufactura, teniendo presente las tolerancias dimensionales de las piezas, que están sometidas a verifica- 
ción metrológica exigente, la cual se realiza en la CMM del Laboratorio de Metrología Dimensional de la Universidad Tecnológica de Pereira.

Conocidas las tolerancias dimensionales de las diferentes piezas y de la estructura final de la CMM, se inicia el proceso de diseño del sistema de visualización de resultados, pasando por el estudio y selección de sensores, finalizando con el desarrollo de software en micro-controlador que integra y manipula los datos generados en los sensores.

Conociendo la secuencia en que se recolectan y analizan los datos generados por los sensores, se elaboran las rutinas y protocolos de calibración de la CMM. El proceso finaliza valorando los resultados generados por la CMM al realizar la calibración utilizando bloques patrón, el cual permite conocer el error e incertidumbre de la CMM, permitiendo establecer las conclusiones y recomendaciones del caso.

\section{METODOLOGÍA Y RESULTADOS}

Una CMM es una máquina de medición de coordenadas que emplea tres componentes móviles que se trasladan a lo largo de guías con recorridos ortogonales. Un operador guía, un palpador alrededor de la pieza para realizar mediciones $(\mathrm{x}, \mathrm{y}, \mathrm{z})$ del tipo: dimensional, posicional, desviaciones geométricas y mediciones de contorno. Los tipos más comunes para las CMM, son: Cantiléver, Puente, Gantry, Brazo Horizontal, Portal, Puente fijo y Brazo articulado (Fig. 1).

Actualmente la industria produce más de $6000 \mathrm{CMM}$ al año (XspectSolutions, 2007). Debido a la gran diversidad de fabricantes, es posible encontrar diferentes configuraciones para cada tipo de CMM (Anujin, 2007) (Fig. 2 y 3). La configuración del bastidor de las CMM depende de la aplicación de las mismas. Dentro de la gran variedad de configuraciones, algunas de ellas sobresalen por su versatilidad como es la tipo puente (Fig. 2d) y cantiléver (Fig. 3f). 


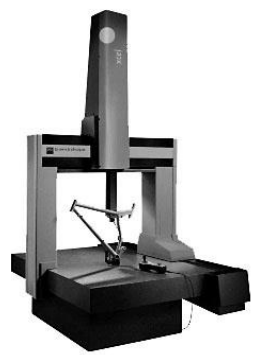

Puente (Bridge)

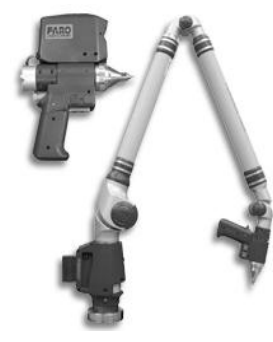

Articuladas

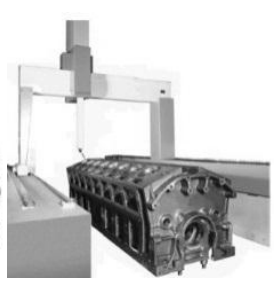

Granty

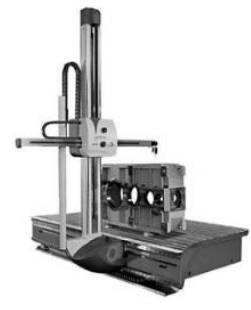

Cantiléver

Fig. 1. Tipos de CMM (Tomada de www.hexagonmetrology.es 10/09/2010)

\subsection{Determinación de la Exactitud Requerida por la CMM}

En el desarrollo de este proyecto, la empresa Suzuki Motor Corporation participó ilustrando el proceso de fabricación y ensamble de motocicletas y suministrando el plano del bastidor de una motocicleta tipo turismo VIVA 115, con el propósito de estudiar la tolerancia dimensional en cada una de las cotas del bastidor y determinar el tipo de calidad ISO.

Los grados de calidad internacional ISO están asociados a los procesos de fabricación industrial, dichos grados identifican las tolerancias permitidas en un proceso de fabricación para una dimensión dada; el sistema ISO prevé más de dieciocho calidades asignadas desde IT01 hasta IT18, siendo IT01 una de las calidades más exigentes e IT18 la calidad más baja (ISO 286-1, 1988).

A cada una de las tolerancias de las dimensiones del bastidor (que van desde $10 \pm 0,5 \mathrm{~mm}$ hasta $1055 \pm 2 \mathrm{~mm}$ ), le fue asignado el correspondiente grado de calidad ISO. Con los valores asignados de calidad, se promediaron y calcularon las medidas estadísticas de la media y la moda, las cuales permiten obtener un valor representativo de la calidad ISO general del bastidor de la motocicleta (Tabla 1).

Tabla 1. Análisis de la Calidad ISO de las medidas de fabricación del bastidor

\begin{tabular}{cc}
\hline Medidas Estadísticas & Calidad ISO (IT) \\
\hline Media & 14 \\
Moda & 16 \\
\hline
\end{tabular}




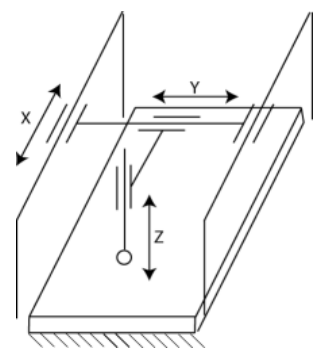

a)

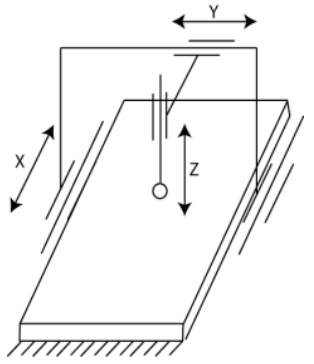

d)

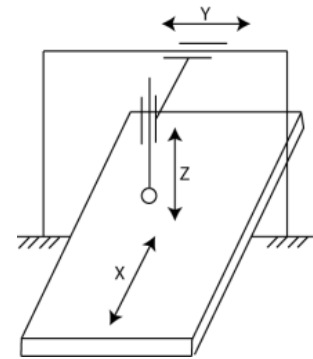

b)

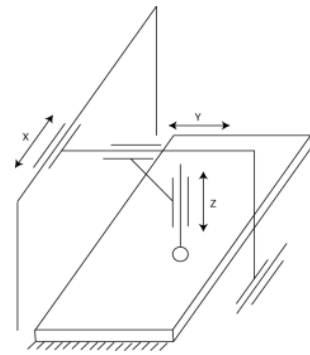

e)

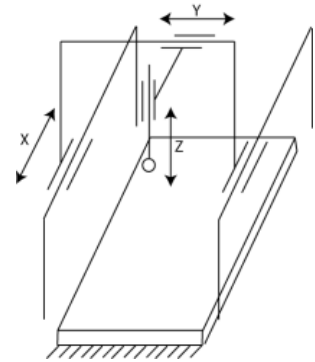

c)

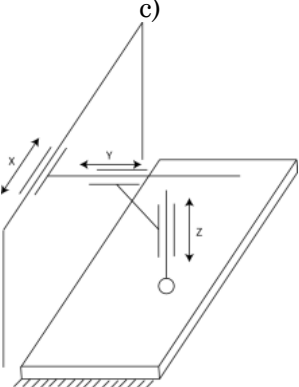

f)

Fig. 2. Configuraciones de las CMM tipo puente

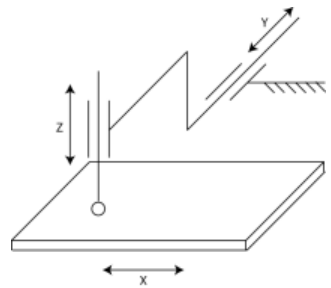

a)

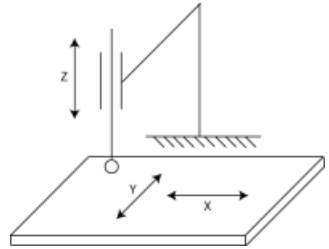

d)

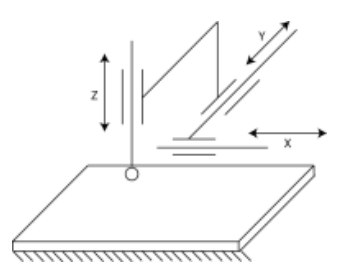

b)

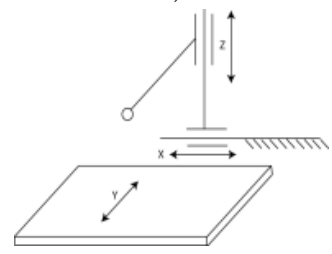

e)

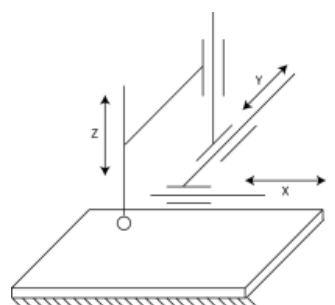

c)

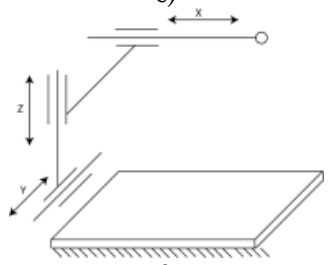

f)

Fig. 3. Configuraciones de las CMM tipo cantiléver 
La clasificación ISO permite asignar conceptos más generales (cualitativos) a la calidad, con el fin de relacionar los valores numéricos con términos de acabado en procesos de manufactura (Tabla 2), obteniéndose un concepto de calidad de acabado basto para el proceso de fabricación del bastidor de la motocicleta.

Tabla 2. Conceptos generales para la clasificación de tolerancias generales para

\begin{tabular}{cc}
\multicolumn{2}{c}{ dimensiones lineales y angulares (ISO 2768-1, 2001) } \\
\hline Calidad ISO (IT) & Concepto sobre la calidad \\
\hline Menor a IT12 & Acabado fino \\
IT12 a IT14 & Acabado medio \\
IT14 a IT16 & Acabado basto \\
Mayor a IT16 & Acabado muy basto \\
\hline
\end{tabular}

La determinación de la calidad ISO de la CMM se obtiene por medio de la tolerancia mínima y la dimensión máxima a medir en el bastidor de motocicleta, teniendo en cuenta que el patrón o dispositivo con el cual se verifica la dimensión de un elemento, debe ser más preciso que el elemento medido (ISO 10012-1, 1997). Según esta información la calidad ISO de la CMM para piezas tipo marco es IT 8, adquirida de la Tabla 3 por medio de una tolerancia requerida de $0,1 \mathrm{~mm}$ y una medida nominal de $1055 \mathrm{~mm}$.

\subsection{Selección de las Características Metrológicas de la CMM}

En la producción de piezas para líneas de ensamble, la calidad del producto (bastidor motocicleta) no sólo depende de la calidad de las máquinas de herramientas usadas para la fabricación; también depende de la exactitud y de la capacidad de repetición de los dispositivos de medición y de inspección (CMM).

Para determinar las características de medición de la CMM se referenciaron dos criterios de selección. El primer criterio de selección fue la determinación del rango mínimo de medición requerido de la CMM. Este rango depende generalmente de las dimensiones de la pieza que se mide; para el caso del bastidor de motocicleta la medida mínima a registrar por la CMM es de $10 \pm$ $0,5 \mathrm{~mm}$. 
Tabla 3. Calidad ISO requerida por la CMM para el control de piezas tipo marco de motocicleta (ISO 10360-2, 2001)

\begin{tabular}{|c|c|c|c|c|c|}
\hline \multicolumn{2}{|c|}{ Medida Nominal (mm) } & \multirow{2}{*}{$\begin{array}{l}\text { Tolerancia } \\
\text { del bastidor } \\
(\mathrm{mm})\end{array}$} & \multirow{2}{*}{$\begin{array}{l}\text { Calidad ISO } \\
\text { del bastidor }\end{array}$} & \multirow{2}{*}{$\begin{array}{l}\text { Tolerancia } \\
\text { requerida } \\
\text { CMM }(\mathrm{mm})\end{array}$} & \multirow{2}{*}{$\begin{array}{l}\text { Calidad ISO } \\
\text { requerida } \\
\text { CMM }\end{array}$} \\
\hline Por encima & $\begin{array}{l}\text { Hasta e } \\
\text { incluido }\end{array}$ & & & & \\
\hline 0 & 3 & 1 & 17 & 0,1 & 12 \\
\hline 3 & 6 & 1 & 17 & 0,1 & 12 \\
\hline 6 & 10 & 1 & 17 & 0,1 & 11 \\
\hline 10 & 18 & 1 & 16 & 0,1 & 11 \\
\hline 18 & 30 & 1 & 16 & 0,1 & 10 \\
\hline 30 & 50 & 1 & 15 & 0,1 & 10 \\
\hline 50 & 80 & 1 & 15 & 0,1 & 10 \\
\hline 80 & 120 & 1 & 15 & 0,1 & 10 \\
\hline 120 & 180 & 1 & 15 & 0,1 & 9 \\
\hline 180 & 250 & 1 & 14 & 0,1 & 9 \\
\hline 250 & 315 & 1 & 14 & 0,1 & 9 \\
\hline 315 & 400 & 1 & 14 & 0,1 & 9 \\
\hline 400 & 500 & 1 & 13 & 0,1 & 8 \\
\hline 800 & 1000 & 1 & 13 & 0,1 & 8 \\
\hline 1000 & 1250 & 1 & 13 & 0,1 & 8 \\
\hline
\end{tabular}

El segundo criterio de selección es la incertidumbre máxima requerida. Las incertidumbres y los métodos de prueba para las CMM se describen en la norma ISO 10360-2 (2001). Para una longitud de medida mínima de $10 \mathrm{~mm}$ y una tolerancia de $\pm 0,5$ determinada en el primer criterio de selección, se obtiene de la Tabla 4 un valor de incertidumbre de 10+L/100. Esto indica que la CMM a construir debe medir valores de tolerancia entre $0,01 \mathrm{~mm}$ y $0,5 \mathrm{~mm}$.

\subsection{Selección del Esquema Cinemático de la CMM}

Para la selección del esquema cinemático de la CMM más adecuado desde el punto de vista de la rigidez, volumen de trabajo y facilidad de operación, se realizaron estudios enfocados en la determinación de la deformación mínima para condiciones iguales de espacio (volumen) de trabajo, fuerza aplicada (para el movimiento del palpador) y material usado en la construcción de la CMM. Este análisis se realizó con la ayuda del software ALGOR, para las 
diferentes configuraciones de las CMM (Fig. 4 y Tabla 5). También se revisaron conceptos como operatividad, mantenimiento y longevidad de las piezas que conformarían la CMM.

Tabla 4. Incertidumbre de las CMM en función de la longitud medida y su tolerancia (ISO 10360-2,2001)

\begin{tabular}{cccccc}
\hline \multirow{2}{*}{ Tolerancia } & \multicolumn{5}{c}{ Longitud de medida $(\mathrm{mm})$} \\
\cline { 2 - 6 } & 10 & 100 & 300 & 600 & 1000 \\
\hline $\pm 0,003 \mathrm{~mm}$ & $0,3+\mathrm{L} / 1000$ & & & & \\
$\pm 0,005 \mathrm{~mm}$ & $0,5+\mathrm{L} / 900$ & $0,4+\mathrm{L} / 1000$ & & & \\
$\pm 0,007 \mathrm{~mm}$ & $0,7+\mathrm{L} / 700$ & $0,6+\mathrm{L} / 900$ & $0,4+\mathrm{L} / 1000$ & & \\
$\pm 0,010 \mathrm{~mm}$ & $1,0+\mathrm{L} / 400$ & $0,8+\mathrm{L} / 500$ & $0,6+\mathrm{L} / 750$ & $0,4+\mathrm{L} / 1000$ & \\
$\pm 0,015 \mathrm{~mm}$ & $1,5+\mathrm{L} / 300$ & $1,2+\mathrm{L} / 300$ & $0,8+\mathrm{L} / 450$ & $0,5+\mathrm{L} / 600$ & $0,5+\mathrm{L} / 1000$ \\
$\pm 0,020 \mathrm{~mm}$ & $2,0+\mathrm{L} / 250$ & $1,6+\mathrm{L} / 250$ & $1,3+\mathrm{L} / 450$ & $0,8+\mathrm{L} / 500$ & $0,8+\mathrm{L} / 800$ \\
$\pm 0,030 \mathrm{~mm}$ & $3,0+\mathrm{L} / 250$ & $2,6+\mathrm{L} / 250$ & $2,0+\mathrm{L} / 300$ & $1,5+\mathrm{L} / 400$ & $1,0+\mathrm{L} / 500$ \\
$\pm 0,050 \mathrm{~mm}$ & $5,0+\mathrm{L} / 150$ & $4,3+\mathrm{L} / 150$ & $3,5+\mathrm{L} / 200$ & $2,6+\mathrm{L} / 250$ & $1,7+\mathrm{L} / 300$ \\
$\pm 0,070 \mathrm{~mm}$ & $7,0+\mathrm{L} / 100$ & $6,0+\mathrm{L} / 100$ & $5,0+\mathrm{L} / 150$ & $4,0+\mathrm{L} / 200$ & $2,0+\mathrm{L} / 200$ \\
$\pm 0.100 \mathrm{~mm}$ & $10+\mathrm{L} / 100$ & $9,0+\mathrm{L} / 100$ & $7,0+\mathrm{L} / 100$ & $6,0+\mathrm{L} / 150$ & $4,0+\mathrm{L} / 150$ \\
\hline
\end{tabular}
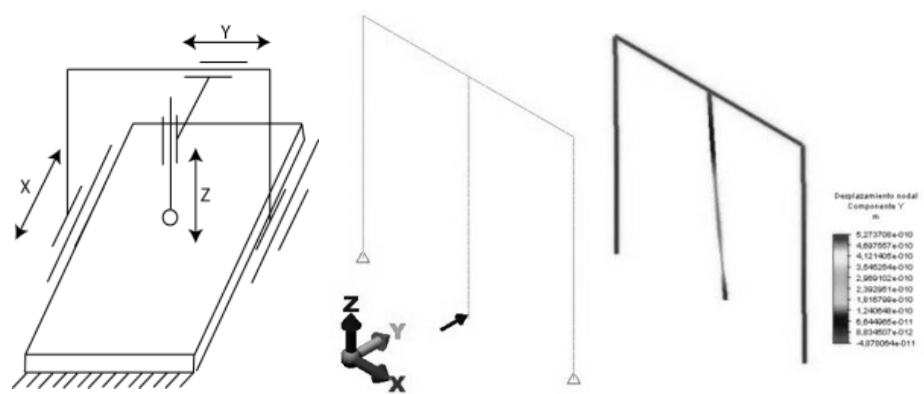

Fig. 4. Representación en ALGOR de un bastidor para estudio de deformación

Tabla 5. Resultados de los análisis de rigidez de bastidores tipo brazo y tipo puente

\begin{tabular}{cccc}
\hline $\begin{array}{c}\text { Nombre } \\
\text { CMM }\end{array}$ & $\begin{array}{c}\text { Desplazamiento } \\
\text { máximo palpador }[\mathrm{m}]\end{array}$ & $\begin{array}{c}\text { Nombre } \\
\text { CMM }\end{array}$ & $\begin{array}{c}\text { Desplazamiento } \\
\text { máximo palpador }[\mathrm{m}]\end{array}$ \\
\hline Figura 2a & $5,302331 \mathrm{e}-10$ & Figura 3a & $1,121954 \mathrm{e}-9$ \\
Figura 2b & $5,396967 \mathrm{e}-10$ & Figura 3b & $1,648549 \mathrm{e}-9$ \\
Figura 2c & $5,321363 \mathrm{e}-10$ & Figura 3c & $1,71037 \mathrm{e}-9$ \\
Figura 2d & $5,273708 \mathrm{e}-10$ & Figura 3d & $1,658541 \mathrm{e}-9$ \\
Figura 2e & $5,18063 \mathrm{e}-10$ & Figura 3e & $4,716903 \mathrm{e}-10$ \\
& & Figura 3g & $1,459931 \mathrm{e}-9$ \\
\hline
\end{tabular}


Al comparar los resultados del análisis, cabe resaltar que el bastidor tipo cantiléver presenta deformaciones $48 \%$ superiores al bastidor tipo puente. Desde el punto de vista operativo, las CMM tipo puente comparadas con las CMM tipo brazo articulado, no requieren de saltos de los puntos de referencia, es decir, no exigen que se cambien las referencias (origen del sistema coordenado) para lograr la verificación metrológica de las piezas tipo marco. Teniendo presentes estos conceptos, la escogencia de la configuración se simplifica a una configuración tipo puente.

\subsection{Determinación de las Tolerancias Geométricas}

Para la determinación de las tolerancias geométricas aplicadas a la construcción del bastidor de una CMM que garanticen la exactitud global de las mediciones realizadas por ellas, se realiza una revisión previa de algunos principios de formación del SAT (Sistema de Ajustes y Tolerancias). El primer principio de formación de SAT establece 20 grados de tolerancia (calidad o grados IT). Se ha determinado que dos o más piezas de distintas medias pueden ser consideradas de igual precisión (que pertenecen a una misma calidad), cuando son elaboradas en la misma maquinaria y bajo unas mismas condiciones de manufactura (régimen de corte, etc.). El segundo principio de formación del SAT establece 27 desviaciones fundamentales para agujeros y 27 desviaciones fundamentales para ejes. El tercero es el sistema de formación de ajustes) y el cuarto la temperatura de medición $\left(+20^{\circ} \mathrm{C}\right)$. Estos dos últimos principios no son relevantes para esta investigación, no son significativas en los cálculos de incertidumbre.

Para el cálculo de las tolerancias requeridas por la CMM, se realizó la síntesis de cadenas dimensionales. El ejercicio básico dimensional en el espacio consiste en el cálculo de la distancia entre dos puntos basados en el cálculo de la magnitud del vector posición. Debido a que la cadena dimensional es corta y la tarea de síntesis reviste complejidad, se analizó el caso de estudio como si se tratara de un problema de análisis. Es decir, se calculó la cadena cinemática asumiendo distintas calidades de los eslabones de entrada para saber cómo cambia la calidad del eslabón de cierre. De esta manera se determinó la calidad de los eslabones de entra- 
da para una calidad conocida del eslabón de cierre. De los aspectos a destacar de la síntesis, sobresale el hecho de que sin importar la calidad de los eslabones de entrada, el eslabón de cierre tiene un grado menor de calidad (según criterio de calidad IT ISO 286-1, 1988). Este concepto permitió predecir la calidad del eslabón de cierre, conociendo las calidades de los eslabones de entrada y viceversa.

\subsection{Realización de Cálculos de Rigidez, Selección de Materiales y Geometría de los Elementos de la CMM}

Para la realización de los cálculos de rigidez, se esbozó un concepto previo de la CMM el cual, con la ayuda del software SolidWorks (para el diseño) y el software ALGOR (para la parte de análisis de ingeniería), orientó el diseño final de la CMM (Fig. 5).
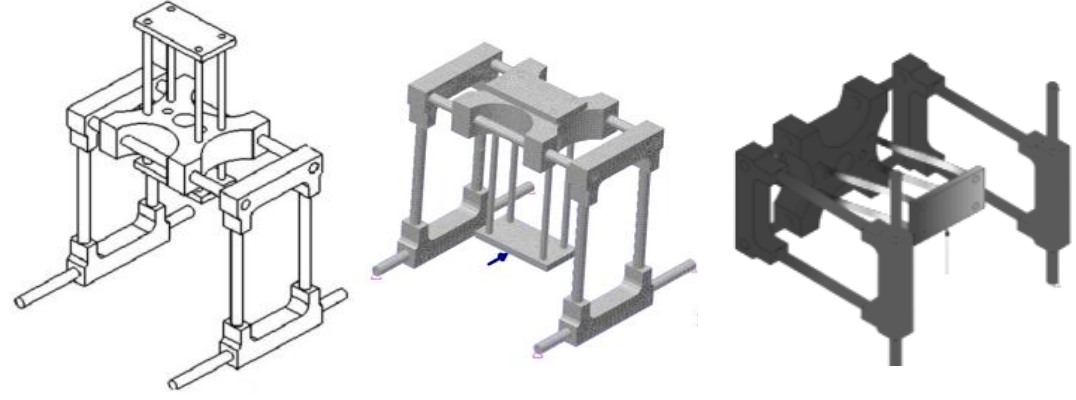

Fig. 5. CAD y CAE de la CMM

Es de resaltar que en la comparación de materiales comerciales, realizada en el proceso de simulación con elementos finitos bajo las mismas condiciones estáticas y dinámicas, las aleaciones de aluminio se deforman un $300 \%$ más que el acero comercial AISI 1020 .

Realizadas las correcciones del caso en la etapa de CAD-CAE, se procede a la fabricación de las piezas utilizando el software Master-CAM (Fig. 6). Este proceso garantiza un control dimensional acorde al exigido por la misma CMM. El control dimensional de las piezas se realizó en la CMM Brown \& Sharpe (Fig. 7), de la Universidad Tecnológica de Pereira. Los resultados de la calibra- 
ción de las piezas resultaron estar acordes a los requeridos para la fabricación de la CMM.
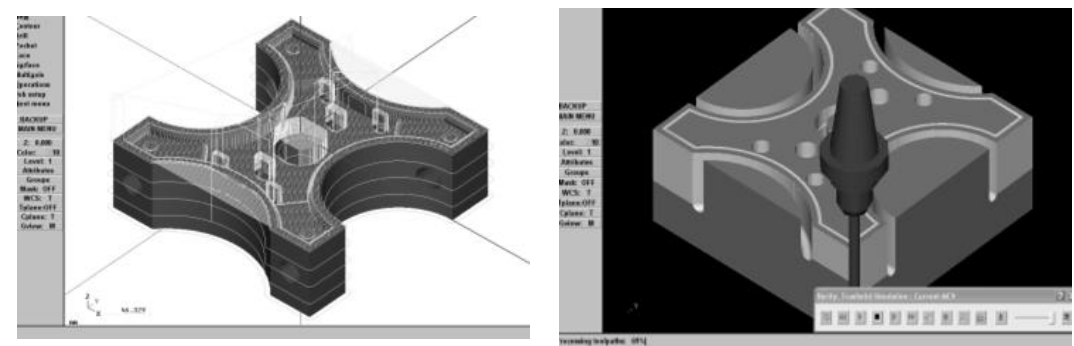

Fig. 6. Mecanizado con la ayuda del software Master-CAM

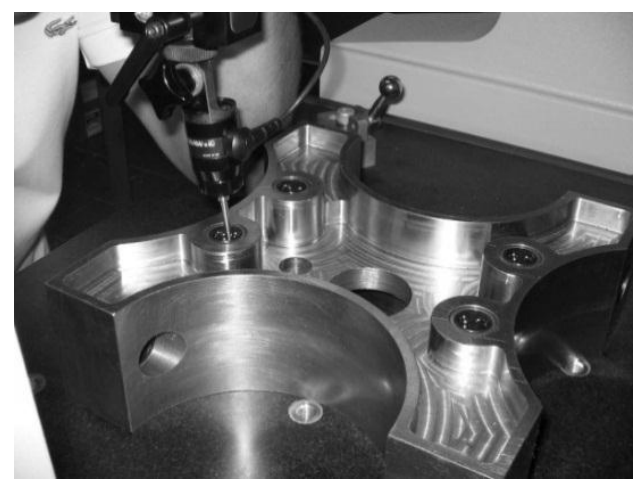

Fig. 7. Verificación dimensional de las piezas usando la CMM Brown \& Sharpe de la Universidad Tecnológica de Pereira

\section{DISEÑO Y CONSTRUCCIÓN DEL SISTEMA DE RECOLECCIÓN DE INFORMACIÓN}

Para conocer la posición espacial del palpador (elemento que realiza el contacto entre la CMM y la pieza verificada), se realizaron pruebas con sensores de posición de infrarrojos, de ultrasonido y sensores de desplazamiento lineal por cable. Conociendo los resultados de las pruebas realizadas a los sensores, se seleccionaron los sensores de desplazamiento lineal por cable, basados en criterios de resolución, repetibilidad y reproducibilidad. Adicional a esto, se diseñó un sistema de adquisición de datos sobre una 
plataforma de un micro controlador Atmega16 programado bajo el lenguaje ANSI C en un ambiente WinAVR (AVRstudio), el cual permite recolectar y manipular la información que brindan los sensores, para luego darle la presentación adecuada en un LCD. Los sensores utilizados son de desplazamiento lineal por cable con salidas digitales de veinticinco pulsos por milímetro $(25 \mathrm{p} / \mathrm{mm})$ (Fig. 8).
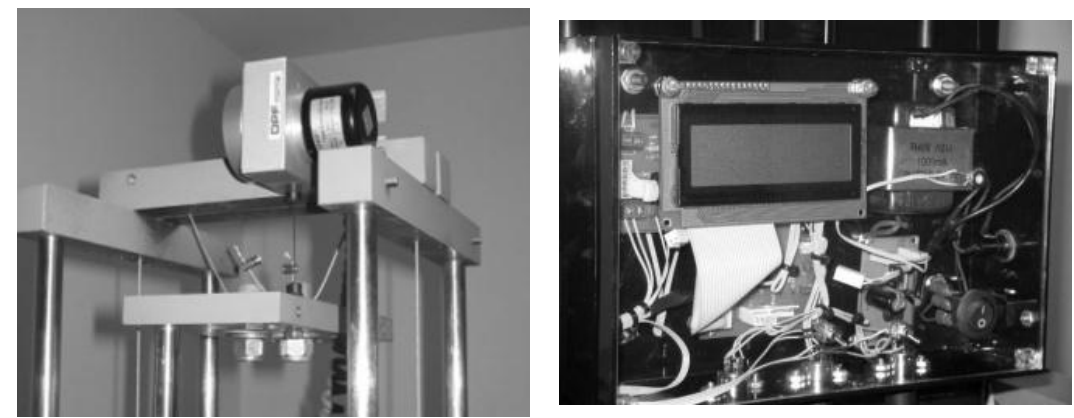

Fig. 8. Montaje de sensores y sistema de visualización

\section{COMPROBACIÓN METROLÓGICA DE LA CMM}

Para la verificación metrológica de la CMM, se utiliza el método tradicional de calibración de instrumentos de medida. El método consiste en usar bloques patrón (galgas) como medida de referencia y comprobar, con mediciones sucesivas, el error y la incertidumbre en la medición reportada por la CMM.

En la verificación de la CMM, el método propuesto está fundamentado en el uso de un caliper checker (Fig. 9). Debido a que la CMM se orientó a la medición de interiores y exteriores, el caliper checker está diseñado de tal forma que se puedan realizar calibraciones de interiores y exteriores en un rango no superior a 300 $\mathrm{mm}$, ofreciendo la facilidad de realizar la verificación de la CMM con un solo montaje.

Este proceso de verificación se realizó bajo condiciones controladas de humedad y temperatura, con el fin de garantizar la estabilidad del patrón (caliper checker). Con un procedimiento de calibración riguroso, se obtuvieron incertidumbres de $1,4 \%$ de la 
medición realizada. Es decir, si la dimensión verificada es de veinte milímetros $(20 \mathrm{~mm})$, la incertidumbre en la medición es de veintiocho milésimas de milímetro $(0,028 \mathrm{~mm})$. Valor que está dentro del rango de tolerancias calculado para este equipo 0,01 $\mathrm{mm}$ y $0,5 \mathrm{~mm}$

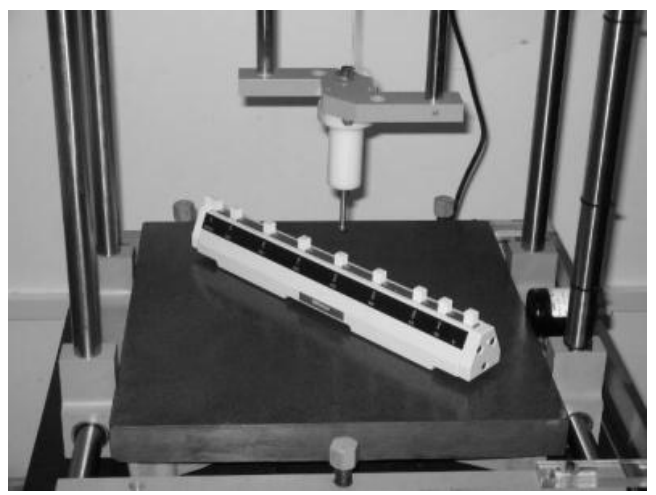

Fig. 9. Verificación de la CMM usando un caliper checker

\section{CONCLUSIONES}

Se diseñó, construyó y fabricó una máquina de medición por coordenadas tipo puente capaz de medir una longitud máxima de $250 \mathrm{~mm}$ con una tolerancia mínima de $\pm 0.3 \mathrm{~mm}$, aplicando conocimientos científicos de manufactura, integrados a procesos $\mathrm{CAD} / \mathrm{CAM} / \mathrm{CAE}$.

Se realizaron modelos teóricos de los diferentes tipos de configuraciones comerciales de las CMM que permitieron la verificación de rigidez de los mismos, obteniéndose que el bastidor tipo puente es un $48 \%$ más rígido que el bastidor tipo brazo.

Se dedujo que la principal dificultad para construir una CMM para la verificación dimensional de piezas tipo bastidor de motocicleta, corresponde a las colosales dimensiones de las piezas que conformarían la CMM. Adicional a esto, se encuentran limitaciones en los procesos de manufactura y verificación para las mismas.

En el proceso de verificación dimensional de las piezas requeridas en la investigación, se comparó el proceso de 
verificación tradicional y de verificación con CMM, encontrándose una reducción de tiempos del 60\%; esta reducción obedece en gran medida al potente software de la CMM, en donde se pueden conocer el diámetro de un agujero y su cilindricidad, el ángulo entre dos planos y la planitud de cada uno de ellos, etc.

Se observaron alternativas para los sensores de desplazamiento como lo fueron el trabajo con sensores de infrarrojos y de ultrasonidos. En ambos casos los resultados fueron satisfactorios (resoluciones en milímetros con sensores de lecturas de centímetros) pero no representativos ante los valores requeridos. Se observó que en el diseño de los componentes electrónicos y el montaje de los sensores, la principal limitante es la consecución.

\section{REFERENCIAS}

Anujin, V.I., (2007); Ajustes y Tolerancias. Editorial Piter, 423.

ISO 10012-1, (1997); Requisitos de aseguramiento de la calidad en los equipos de medida - Parte 1: Sistema de confirmación metrológica de los equipos de medida.

ISO 10360-1, (2000); Geometrical Product Specifications (GPS) Acceptance and reverification tests for coordinate measuring machines (CMM), 3 p.

ISO 10360-2, (2001); Geometrical Product Specifications (GPS) Acceptance and reverification tests for coordinate measuring machines (CMM), $20 \mathrm{p}$.

ISO 2768-1, (2001); General Tolerances - Part 1: Tolerances for linear and angular dimensions without individual tolerance indication.

ISO 286-1, (1988); Sistema ISO de tolerancias y ajustes Parte 1: Base de tolerancias, desviaciones y ajustes, $52 \mathrm{p}$.

XspectSolution, (2007); XspectSolutions, Recuperado el 12 de 10 de 2009, XspectSolutions:http://www.xpectsolutions.com/CMM-History.aspx. 
